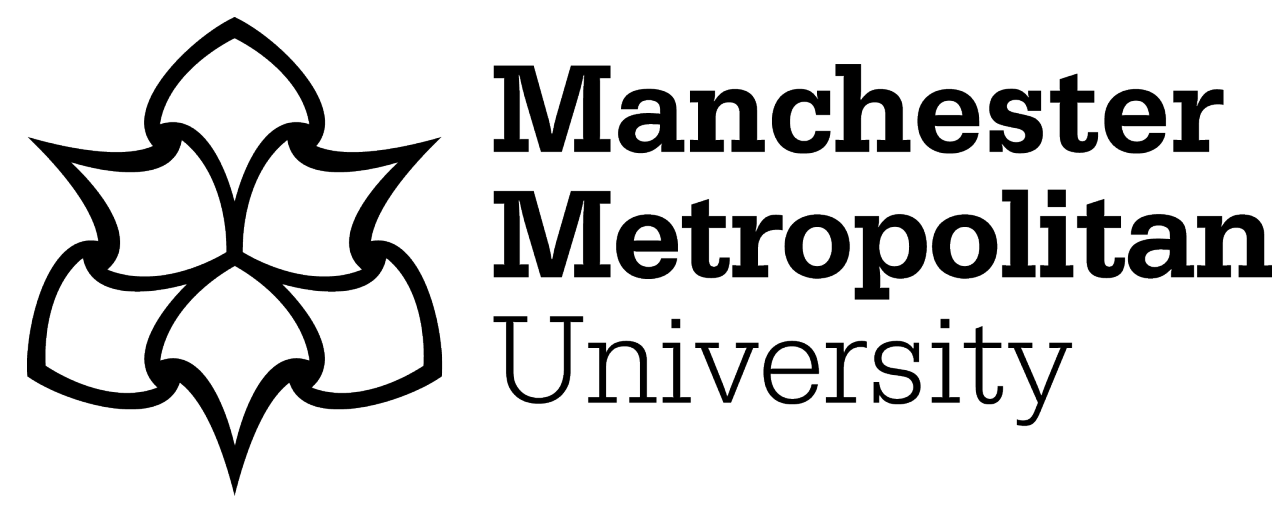

King, LA ORCID logoORCID: https://orcid.org/0000-0002-0772-2378 and Parkinson, BA (2016) Photosensitization of ZnO Crystals with lodide-Capped PbSe Quantum Dots. Journal of Physical Chemistry Letters, 7 (14). pp. 2844-2848. ISSN 1948-7185

Downloaded from: https://e-space.mmu.ac.uk/624301/

Version: Accepted Version

Publisher: American Chemical Society (ACS)

DOI: https://doi.org/10.1021/acs.jpclett.6b01133

Please cite the published version 


\title{
Photosensitization of ZnO Crystals with Iodide Capped PbSe Quantum Dots
}

\author{
Laurie A. King ${ }^{1}$ and B. A. Parkinson ${ }^{1}$ \\ ${ }^{1}$ Department of Chemistry and School of Energy Resources \\ University of Wyoming \\ 1000 E University \\ Laramie, WY 82071
}

\begin{abstract}
Lead selenide (PbSe) quantum dots (QDs) are an attractive material for application in photovoltaic devices due to the ability to tune their band gap, efficient multiple exciton generation and high extinction coefficients. However, PbSe QDs are quite unstable to oxidation in air. Recently there have been multiple studies detailing post-synthetic halide treatments to stabilize lead chalcogenide QDs. We exploit iodide stabilized PbSe QDs in a model quantum dot sensitized solar cell configuration where zinc oxide ( $\mathrm{ZnO}$ ) single crystals are sensitized using cysteine as a bifunctional linker molecule. Sensitized photocurrents stable for $>1$ hour can be measured in aqueous KI electrolyte that is usually corrosive to QDs under illumination. The spectral response of the sensitization extended out to $1700 \mathrm{~nm}$, the farthest into the infrared yet observed. Hints of the existence of multiple exciton generation and collection as photocurrent, as would be expected in this system, are speculated and discussed.
\end{abstract}

\section{TOC Graphic}

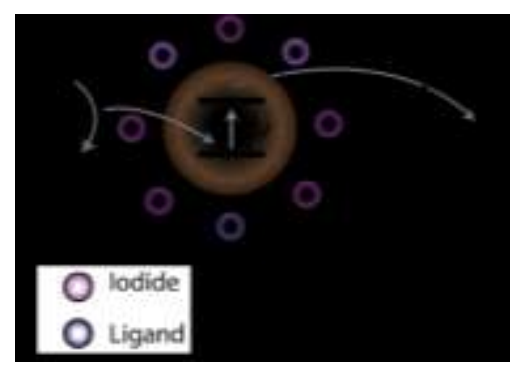

\section{Key words}

Quantum-dot sensitized solar cells (QDSSC), metal oxide single crystal sensitization, lead selenide quantum dots, $\mathrm{PbSe}$ 
Due to their tuneable band gaps, high extinction coefficients, and the ability to inject hot electrons and multiple exciton generation (MEG), ${ }^{1}$ lead chalcogenide quantum dots (QDs) are considered promising candidates for photovoltaic, and optoelectronic applications. ${ }^{2}$ Amongst other applications, inorganic semiconducting QDs have been often investigated as replacements for dyes in dye-sensitized solar cells (quantum dot sensitized solar cells, QDSSCs) and as flurophores in display applications. ${ }^{3}$

Lead selenide (PbSe) QDs are exceptionally attractive materials for IR-sensitive solar cell and photodetector applications given their small bulk band gap of $0.28 \mathrm{eV}$ that can be increased up to $0.8 \mathrm{eV}$ from quantum confinement, large Bohr radius ( $46 \mathrm{~nm}$ ) and quite small electron and hole effective masses. ${ }^{4}$ In particular, colloidal PbSe QDs are known for highly efficient multiple exciton generation. ${ }^{4-6}$ Despite the appropriate intrinsic properties of $\mathrm{PbSe}$ QDs, air stability has remained an Achilles heal for applications. Indeed, it has been reported that air exposure leads to oxidation of up to $50 \%$ of both $\mathrm{Pb}$ and Se atoms within 24 hours 7 and leads to a reduction in the size of the PbSe core with a resulting blue shift of the optical absorption and emission. ${ }^{7}$ Various strategies have been investigated to stabilize PbSe and $\mathrm{PbS}$ QDs. One strategy to prevent air oxidation has been to grow core/shell structures (e.g. $\mathrm{PbSe} / \mathrm{ZnSe},{ }^{8} \mathrm{PbSe} / \mathrm{PbS},{ }^{9} \mathrm{PbSe} / \mathrm{CdSe}^{10,7}$ ) where the outer, more stable shell material protects the PbSe core from oxidation. Atomic layer deposition (ALD) of protective layers on top of QDs has also been explored to enhance stability as well as carrier mobilities. ${ }^{11,12}$ Alternative strategies have explored the replacement of hydrophobic surfactant ligands with allinorganic ligands, such as molecular metal chalcogenides (MMC) like $\mathrm{SnS}_{4}^{4-.13}$ Recently, passivation by halide shells has been reported to protect both $\mathrm{PbS} 14,15,16$ and $\mathrm{PbSe}{ }^{17,18,19} \mathrm{QDs}$ from air oxidation for up to several months. Both solid state and liquid phase halide ligand exchanges on metal chalcogenide QDs have been demonstrated and shown to preserve both the optical properties as well as the electronic structure of QDs.

In this letter we utilize iodide capped PbSe QDs (PbSe-I) in a model QDSSC configuration. Furthermore, we employ a linker-assisted attachment to a zinc oxide (ZnO) single crystal surface using protonated cysteine to provide an electrostatic attraction to the negatively charged, air exposed iodide capped PbSe QDs. ZnO single crystals, a transparent wide band gap metal oxide semiconductor, permit back-side illumination that is necessary to probe far into the NIR to avoid competitive light absorption from the aqueous electrolyte solution. Single crystals also enable morphological studies (AFM) of the QD attachment that would otherwise be inaccessible in a mesoporous morphology.20,21 We demonstrate 
sensitization of a semiconductor electrode farther into the NIR than any yet reported as well as the first use of iodide capped PbSe in a QDSSC architecture in a liquid electrolyte. ${ }^{22}$

A TEM micrograph of the as-synthesized PbSe QDs with an average diameter of 6.5 $\mathrm{nm}$ is shown in Figure 1A. The UV-Vis absorbance spectrum of the iodide capped QDs shows a distinct first excitonic peak at approximately $1700 \mathrm{~nm}(0.73 \mathrm{eV})$. XPS spectra (Figure S1) provide evidence for the successful iodide capping of the PbSe QDs. As reported by Ning et al.,15 the ligand exchange does not replace all of the as synthesised oleic acid ligands which remain at the surface of the QDs.

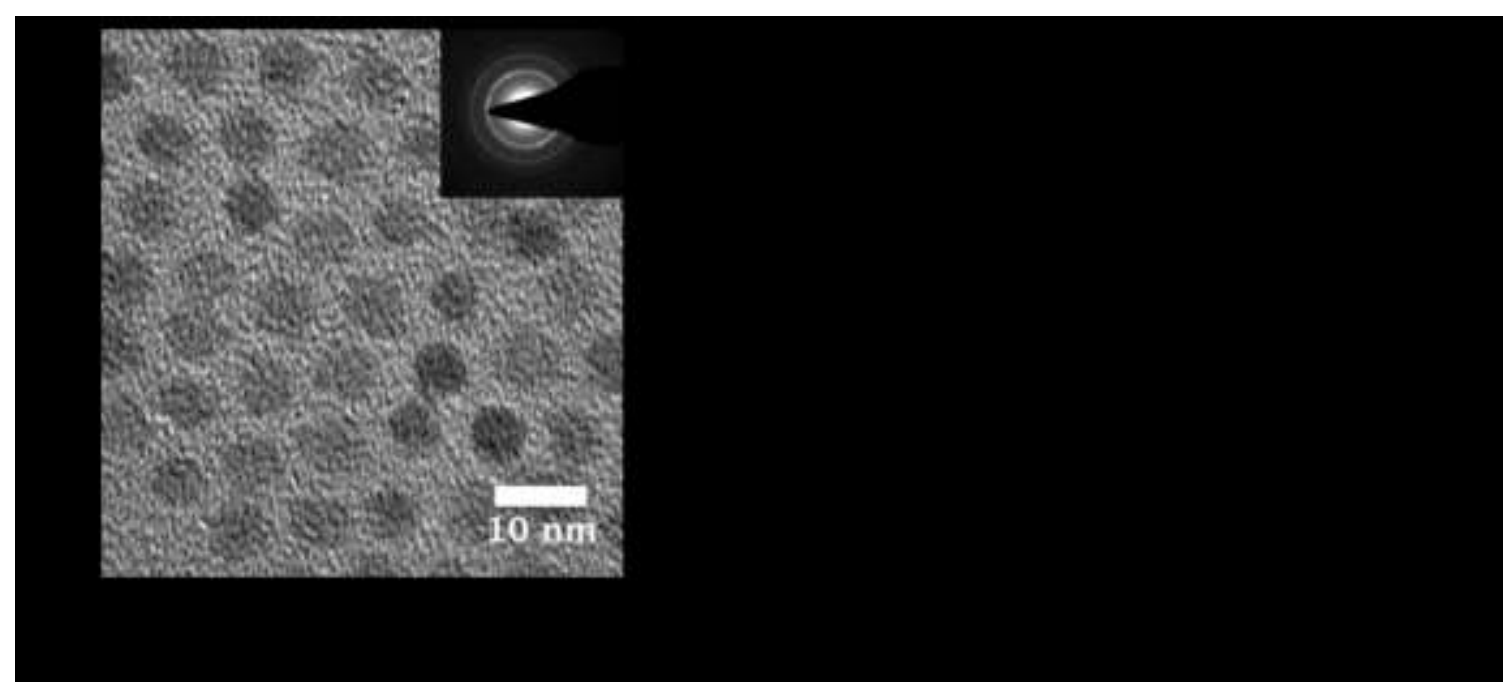

Figure 1. A. TEM micrograph of the as synthesised oleic acid $(\mathrm{OA})$ capped PbSe QDs. The insert is a selected area electron diffraction pattern (SAED) of the QDs demonstrating the crystalline structure of the PbSe QDs. B. UV-vis absorption spectra of the PbSe-OA QD sol suspended in TCE. The inset is an expanded scale of the N-IR region of the spectrum showing the first exciton peak of the QDs.

Linker molecules with multiple functional groups, such as thiols, amines, and carboxylic acids, are often used for attaching QDs to a wide band gap metal oxide surfaces. Cysteine, having a thiol, amine and carboxylic acid functional groups, is thus an attractive linker molecule for facilitating QD surface attachment. Indeed, cysteine has been shown to enhance IPCE's for CdSe QDSSCs relative to the commonly used mercapto-alkonic acids. ${ }^{23}$ In this work we use cysteine when both the thiol and amine are protonated such that the electrostatic interaction between the positively charged cysteine and the negatively charged iodide capping ligands on the PbSe will bridge to the $\mathrm{ZnO}$ surface. The $\mathrm{pK}_{\mathrm{a}}$ of the thiol, amine and carboxylic acid groups of cysteine are 8.14, 10.28 and 1.91 respectively. ${ }^{24}$ Therefore, functionalizing a $\mathrm{ZnO}$ crystal surface with a pH 3.9 cysteine solution results in the thiol and amine groups both being protonated and the carboxylic acid is deprotonated. 


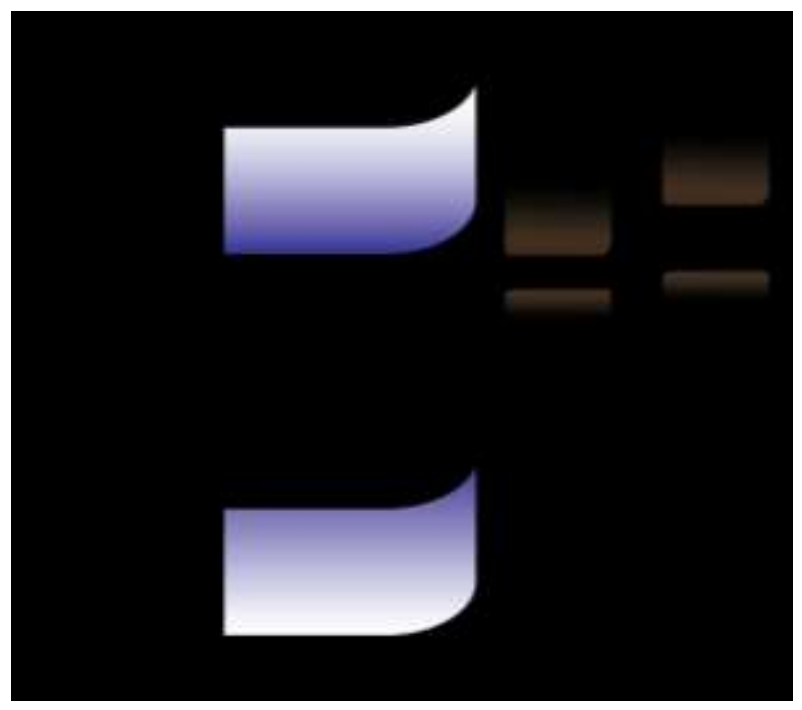

Figure 2. Band energy level diagram depicting the relevant energy levels of bulk PbSe and $6.5 \mathrm{~nm}$ $\mathrm{PbSe} \mathrm{QDs}$ and the ZnO crystal. The energy positions for PbSe were calculated from reference 27.

The AFM images of clean ZnO (A) and PbSe QD sensitized ZnO (B) surfaces are shown in Figure 3. The clean terraced surface is distinctive with curved terraces and non-uniform terrace widths ranging from 20 - $500 \mathrm{~nm}$. Both single isolated QDs (approximately $8 \mathrm{~nm}$ high, slightly higher than the measured TEM size due to capping iodide ligands) and QD agglomerates (10 - $40 \mathrm{~nm}$ high) were observed on the QD sensitized surface. Example AFM line traces are shown in Figure S2. Image analysis of the AFM data revealed approximately $20 \%$ QD surface coverage (see SI for details of the calculation).

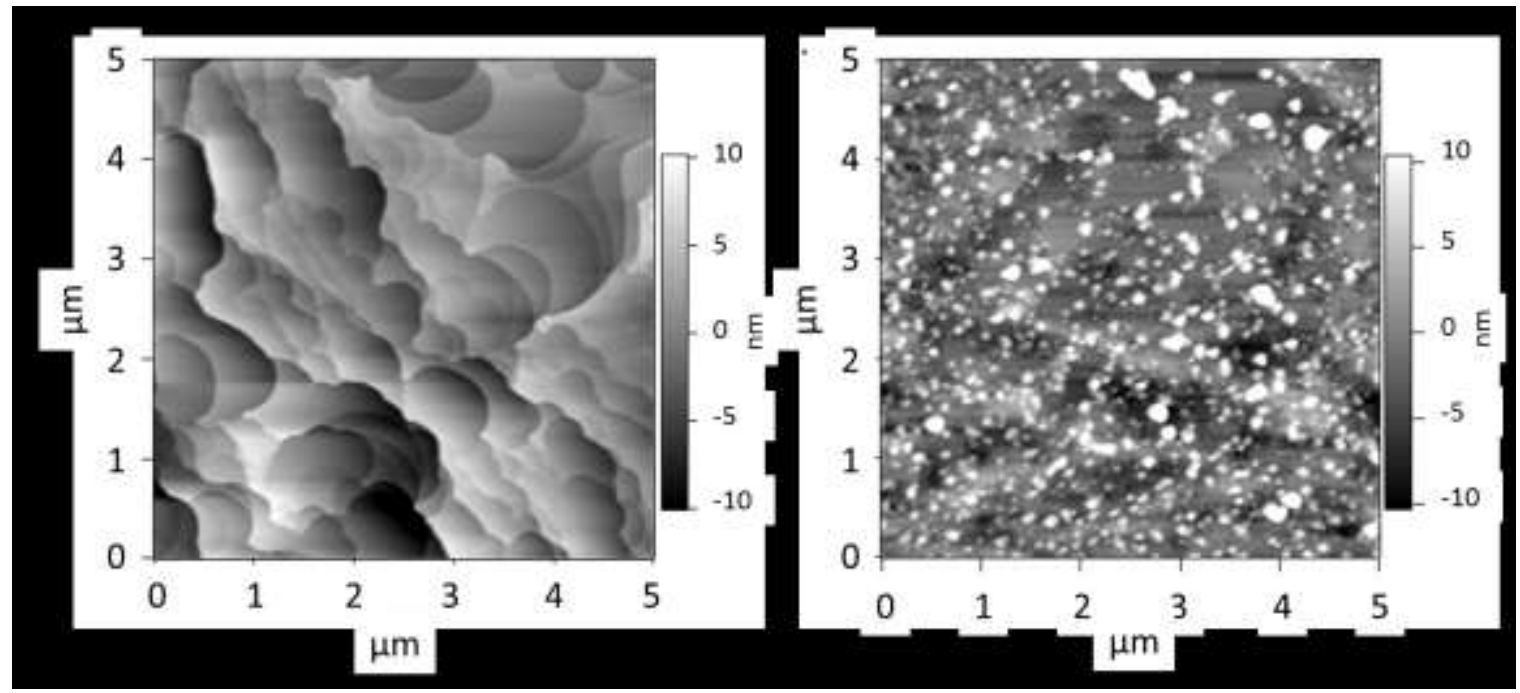


Figure 3. AFM images of A. a clean as prepared (0001) Zn-face ZnO crystal, and; B. Cysteine and PbSe QD modified ZnO (0001) Zn-face surface.

The incident photon conversion efficiency (IPCE) spectrum for a PbSe QD sensitized $\mathrm{ZnO}$ crystal is shown in Figure 4. Aqueous $\mathrm{KI}$ electrolyte solution was used as a regenerating redox couple for photocurrent measurements and all measurements are taken with backside illumination configuration. Iodide based redox couples are typically used in dye sensitized solar cells (DSSCs) (due to its slow recombination kinetics at the oxide semiconductor surface ${ }^{25}$ but have not been used with QDSSCs since inorganic QDs are unstable to triiodide oxidation. ${ }^{14,26}$ In this work, given the QD capping agent is iodide, KI was used as the redox mediator. Aqueous KI is known to photocorrode CdSe QDs. Specifically, prolonged exposure to monochromatic light was shown to lead to a blue-shift in the first excitonic peak and was accredited to a shrinkage of the CdSe QDs to the size whereby the band gap exceeds the monochromatic excitation energy. Despite such instabilities, coupled with the inherent air oxidation (instability) of lead chalcogenide QDs, we successfully measure IPCE signal (Figure 4). Indeed, Figure 4 shows that photoexcited electron injection from the first excitonic peak $(1700 \mathrm{~nm})$ is clearly measured in the IPCE spectrum as expected from the appropriate band alignment between the first excited state of the $6.5 \mathrm{~nm}$ PbSe QDs and $\mathrm{ZnO}$ conduction band (Figure 2). The much smaller background IPCE spectrum for the same unsensitized ZnO single crystal is also shown in Figure 4 (green points). To the best of our knowledge, 1700 $\mathrm{nm}$ is the furthest red sensitization reported for any sensitized photoelectrode, being further into the IR than our previous report of $\mathrm{PbS}$ sensitization of $\mathrm{ZnO}$ out to $1580 \mathrm{~nm} .{ }^{22}$ The light harvesting efficiency (LHE) of the QD solution used to sensitize the $\mathrm{ZnO}$ and normalized to the exciton peak at $1700 \mathrm{~nm}$ is also shown in Figure 4. Details of how the LHE was calculated are provided in the supporting information. 


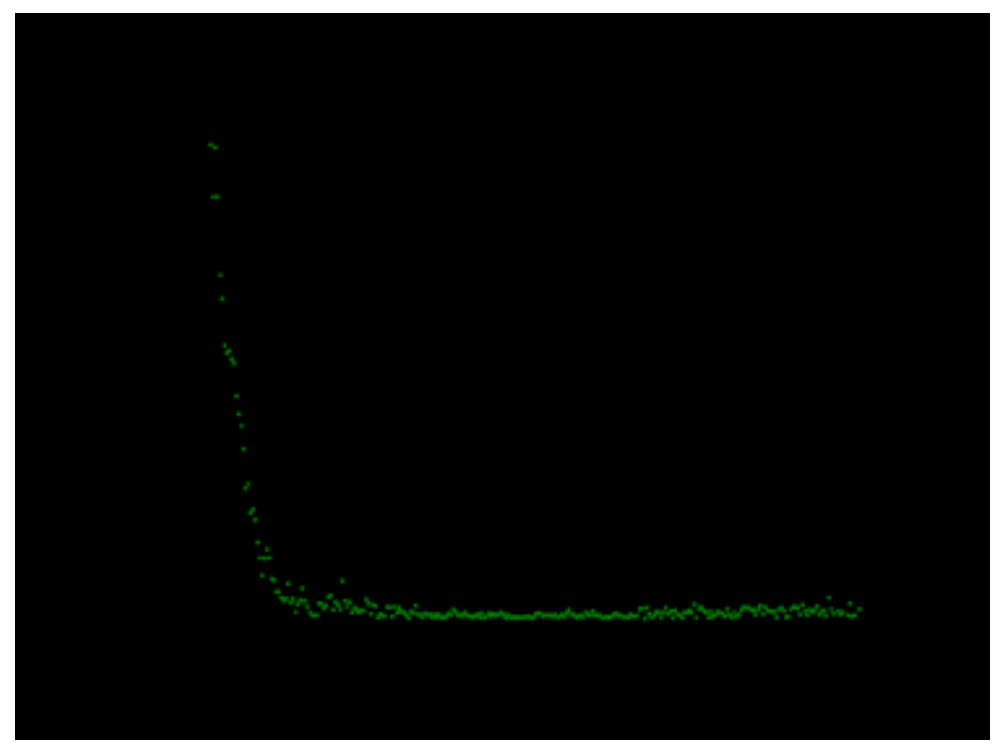

Figure 4. The IPCE spectra of a PbSe sensitized ZnO (black dots) and an unsensitized ZnO crystal (green dots) collected at 0 V vs. Pt wire bias. The light harvesting efficiency (LHE) of the QD sol used to sensitize the $\mathrm{ZnO}$ is overlaid for comparison (dashed line).

The photocurrent spectrum begins to show a positive deviation from the LHE at about $900 \mathrm{~nm}$, near where extra electrons might be injected from MEG in the QDs since it is approximately $\sim 2 \times \mathrm{Eg}$ (band gap) of the adsorbed QDs. However, the deviation is not a factor of 2 that would be expected from an efficient MEG collection from all adsorbed QDs if they were strongly electronically coupled to the $\mathrm{ZnO}$ substrate as in our previous work on PbS QDs on anatase $\mathrm{TiO}_{2} .^{1}$ The deviation becomes much larger at about $550 \mathrm{~nm}$ or $\sim 3 \mathrm{x}$ Eg, indicating we may actually be seeing 3 electrons per photon in this experiment but the background IPCE from the sub-bandgap states of the $\mathrm{ZnO}$ substrate is also increasing at these energies. Although it is quite possible given the photophysics of the system, ${ }^{4}$ we cannot make any definitive claims about achieving the collection of 2 or 3 electrons per photon in this system. This is due to several unknowns, including that the LHE of the adsorbed QDs on the ZnO crystal has not been measured and there is aggregation of QDs on the $\mathrm{ZnO}$ surface (Figure 3) and presumably not in solution the actual LHE of the surface bound QDs could be different. The less than a factor of 2 increase in the IPCE compared to the LHE in solution might also indicate that all QDs in clusters are not well electronically coupled to the $\mathrm{ZnO}$. Also the iodide capping groups that stabilize the PbSe QDs may not be effective at electronically coupling to the $\mathrm{ZnO}$ conduction band states through the surface bound cysteine molecules. 
Repeated measurements of the IPCE spectra are shown in Figure S4 as a function of time. The stability of these photoanodes was found to vary from sample to sample (all made from the same QD batch). However, for each sample tested, sensitized photocurrents remained mostly stable for over 1 hour of repeated scanning IPCE spectra. We postulate that the instability is due to the incomplete ligand exchange to iodide, leading to the oxidation and/or desorption of the PbSe. Future work will be conducted to investigate the influence of a complete shell on the direct exposure to electrolyte.

\begin{tabular}{lccc}
\hline Element & \multicolumn{2}{l}{$\begin{array}{l}\text { Binding } \\
\text { Energy (eV) }\end{array}$} & $\begin{array}{l}\text { Atomic } \\
\text { Percentage (\%) } \\
\text { Befor }\end{array}$ \\
& \multicolumn{3}{l}{ efter } \\
Pb 4f & 137 & 8 & 7 \\
I 3d & 617 & 2 & 2 \\
Se 3d & 46 & 6 & 3 \\
O 1s & 528 & 61 & 57 \\
Zn 2p & 1019 & 23 & 31 \\
\hline
\end{tabular}

Table 1. XPS atomic percentages for samples before and after exposure to aqueous KI electrolyte.

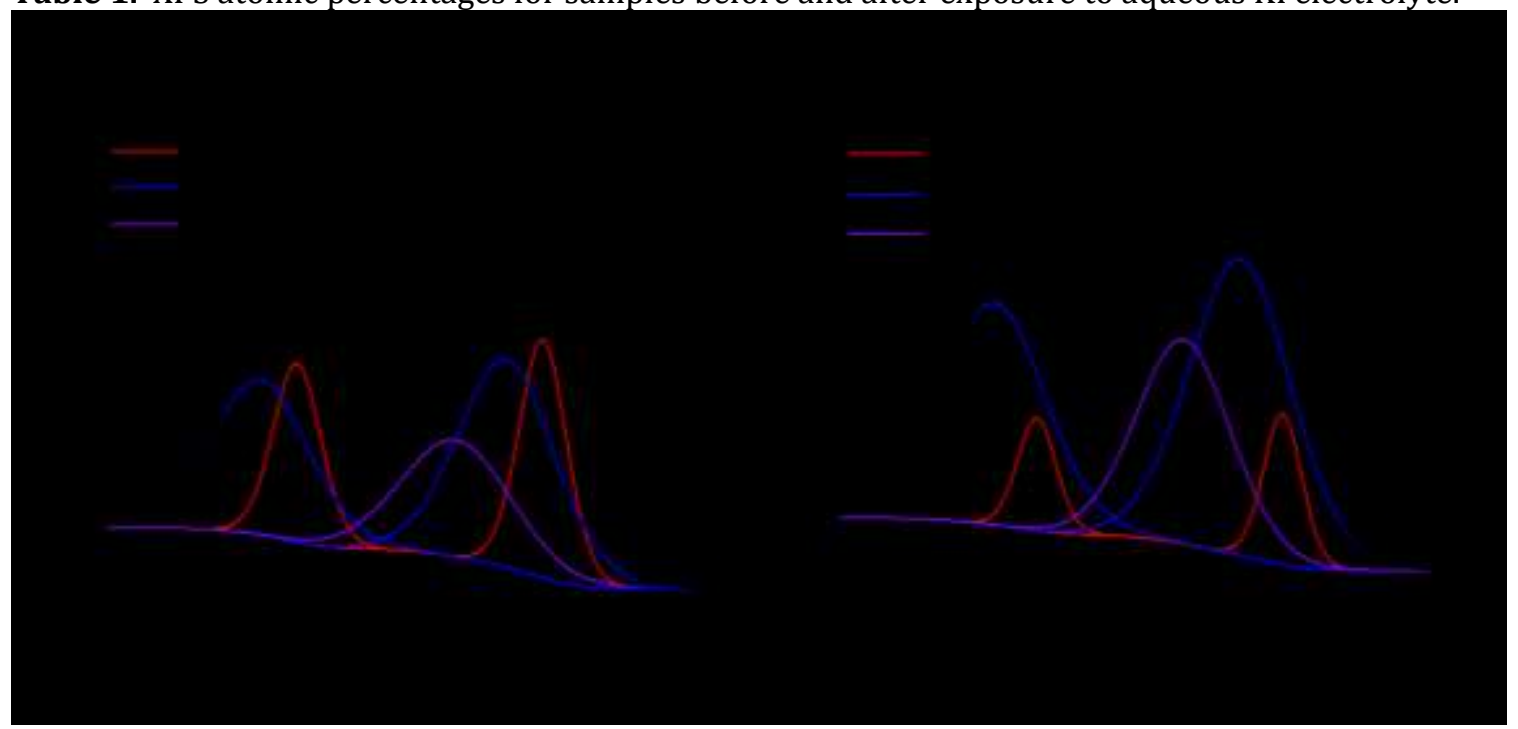

Figure 5. XPS spectra of the $\mathrm{Pb} 4 \mathrm{f}$ region for $\mathrm{PbSe}$ sensitized $\mathrm{ZnO}$ single crystal, (A) before IPCE measurement and (B) after IPCE measurement.

XPS was performed on the $\mathrm{ZnO}$ crystals to characterize the composition and chemical state of the PbSe QDs before and after photoelectrochemical experiments. The atomic percentages from XPS (calculated from the survey scan) are shown in Table 1. These data show that the atomic percentages of lead, iodide and oxygen remain relatively constant 
during IPCE measurements, however the zinc atomic percent increases and the selenium signal decreases. The presence of iodide both before and after photoelectrochemistry implies that the PbSe remains capped with iodide (Figure $\mathrm{S} 2$ ). The $\mathrm{Pb} 4 \mathrm{f}$ high resolution XPS data is shown in Figure 5. Significant overlap with the Zn 3s peaks is observed in this region. In agreement with the XPS survey scan, the relative contribution from the $\mathrm{Zn} 3 \mathrm{~s}$ peak increases, implying a decrease in the total QD population adsorbed at the surface. There exists clear peak splitting of the $\mathrm{Pb} 4 \mathrm{f}$ peak in the XPS spectra both before and after electrolyte exposure suggesting the presence of lead oxide $(138.5 \mathrm{eV}) .{ }^{19}$ The contribution from the oxidative peak is enhanced post exposure suggesting an increase in the extent of lead oxidation. Given the $\sim 50 \mathrm{~nm}$ blue shift observed in the IPCE data over repeat runs (Figure S4), relative to the absorbance spectra of the as-synthesised PbSe QDs, the XPS data verifies a slight decrease in PbSe QD size, presumably due to oxidation of lead. Using the size dependent extinction coefficient of PbSe QDs from Moreels et al., the estimated change in core diameter is $0.5 \mathrm{~nm}$, which is approximately one unit cell of $\mathrm{PbSe} .^{27}$

In conclusion, we have demonstrated the use of iodide capped PbSe QDs as a sensitizer in a QDSSC configuration. Protonated cysteine was used as an electron coupling ligand to promote sensitization at the $\mathrm{ZnO}$ single crystal surface. To the best of our knowledge, this study reports the furthest N-IR sensitization of $>1650 \mathrm{~nm}$ from a first exciton injection. We speculate the relative stability of our device is a consequence of the iodide ligands that inhibit the oxidation of the PbSe QDs. Hints of multiple exciton generation and collection are inferred from comparing the LHE of a PbSE QD sensitizing solution to the IPCE spectra where there is a significant positive deviation from the IPCE spectra at blue wavelengths.

\section{Acknowledgement}

The authors wish to acknowledge the U.S. Department of Energy, Office of Science, Basic Energy Sciences, Division of Chemical Sciences, Geosciences and Biosciences for financial support through grant DE-FG03-96ER14625. The authors would also like to thank Dr. Erwin Sabio for his help with TEM and XPS analysis.

\section{Associated Content \\ Supporting Information}


Experimental methods (synthesis of QDs, ligand exchange, sensitization and photoelectrochemical measurements/proceedures) are provided in the supporting information. Additional information and results including AFM line scans, light harvesting efficiency (LHE) calculations and XPS data (iodide) of pre and post IPCE measurements are also provided in the supporting information. This information is available free of charge on the ACS Publications website at DOI:

\section{References}

(1) Sambur, J. B.; Novet, T.; Parkinson, B. A. Multiple Exciton Collection in a Sensitized Photovoltaic System. Science 2010, 330, 63-66.

(2) Bang, J. H.; Kamat, P. V. Quantum Dot Sensitized Solar Cells. A Tale of Two Semiconductor Nanocrystals: CdSe and CdTe. Acs Nano 2009, 3, 1467-1476.

(3) Kamat, P. V. Quantum Dot Solar Cells. Semiconductor Nanocrystals as Light Harvesters. J. Phys. Chem. C 2008, 112, 18737-18753.

(4) Ellingson, R. J.; Beard, M. C.; Johnson, J. C.; Yu, P. R.; Micic, O. I.; Nozik, A. J.; Shabaev, A.; Efros, A. L. Highly Efficient Multiple Exciton Generation in Colloidal PbSe and PbS Quantum Dots. Nano Lett. 2005, 5, 865-871.

(5) Luther, J. M.; Beard, M. C.; Song, Q.; Law, M.; Ellingson, R. J.; Nozik, A. J. Multiple Exciton Generation in Films of Electronically Coupled PbSe Quantum Dots. Nano Lett. 2007, 7, 1779-1784.

(6) Midgett, A. G.; Luther, J. M.; Stewart, J. T.; Smith, D. K.; Padilha, L. A.; Klimov, V. I.; Nozik, A. J.; Beard, M. C. Size and Composition Dependent Multiple Exciton Generation Efficiency in $\mathrm{PbS}, \mathrm{PbSe}$, and $\mathrm{PbS}_{\mathrm{x}} \mathrm{Se}_{1-\mathrm{x}}$ Alloyed Quantum Dots. Nano Lett. 2013, 13, 3078-3085.

(7) Sykora, M.; Koposov, A. Y.; McGuire, J. A.; Schulze, R. K.; Tretiak, O.; Pietryga, J. M.; Klimov, V. I. Effect of Air Exposure on Surface Properties, Electronic Structure, and Carrier Relaxation in PbSe Nanocrystals. ACS Nano 2010, 4 , 2021-2034.

(8) Kim, S.; Marshall, A. R.; Kroupa, D. M.; Miller, E. M.; Luther, J. M.; Jeong, S.; Beard, M. C. Air-Stable and Efficient PbSe Quantum-Dot Solar Cells Based upon ZnSe to PbSe Cation-Exchanged Quantum Dots. ACS Nano 2015, 9, 81578164.

(9) Yanover, D.; Vaxenburg, R.; Tilchin, J.; Rubin-Brusilovski, A.; Zaiats, G.; Čapek, R. K.; Sashchiuk, A.; Lifshitz, E. Significance of Small-Sized PbSe/PbS Core/Shell Colloidal Quantum Dots for Optoelectronic Applications. J. Phys. Chem. C 2014, 118, 17001-17009.

(10) Pietryga, J. M.; Werder, D. J.; Williams, D. J.; Casson, J. L.; Schaller, R. D.; Klimov, V. I.; Hollingsworth, J. A. Utilizing the Lability of Lead Selenide to Produce Heterostructured Nanocrystals with Bright, Stable Infrared Emission. J. Am. Chem. Soc. 2008, 130, 4879-4885. 
(11) ten Cate, S.; Liu, Y.; Suchand Sandeep, C. S.; Kinge, S.; Houtepen, A. J.; Savenije, T. J.; Schins, J. M.; Law, M.; Siebbeles, L. D. A. Activating Carrier Multiplication in PbSe Quantum Dot Solids by Infilling with Atomic Layer Deposition. J. Phys. Chem. Lett. 2013, 4, 1766-1770.

(12) Palmstrom, A. F.; Santra, P. K.; Bent, S. F. Atomic Layer Deposition in Nanostructured Photovoltaics: Tuning Optical, Electronic and Surface Properties. Nanoscale 2015, 7, 12266-12283.

(13) Jin, H.; Choi, S.; Xing, G.; Lee, J.-H.; Kwon, Y.; Chong, W. K.; Sum, T. C.; Jang, H. M.; Kim, S. SnS4 ${ }^{4-}{ }^{4} \mathrm{SbS}_{4}{ }^{3-}$, and $\mathrm{AsS}_{3}{ }^{3-}$ Metal Chalcogenide Surface Ligands: Couplings to Quantum Dots, Electron Transfers, and All-Inorganic Multilayered Quantum Dot Sensitized Solar Cells. J. Am. Chem. Soc. 2015, 137, 13827-13835.

(14) Niu, G.; Wang, L.; Gao, R.; Ma, B.; Dong, H.; Qiu, Y. Inorganic Iodide Ligands in Ex Situ PbS Quantum Dot Sensitized Solar Cells with $\mathrm{I}^{-} / \mathrm{I}^{-}$Electrolytes. J. Mater. Chem. 2012, 22, 16914-16919.

(15) Ning, Z.; Voznyy, O.; Pan, J.; Hoogland, S.; Adinolfi, V.; Xu, J.; Li, M.; Kirmani, A. R.; Sun, J.-P.; Minor, J.; Kemp, K. W.; Dong, H.; Rollny, L.; Labelle, A.; Carey, G.; Sutherland, B.; Hill, I.; Amassian, A.; Liu, H.; Tang, J.; Bakr, O. M.; Sargent, E. H. Air-Stable N-Type Colloidal Quantum Dot Solids. Nat. Mater. 2014, 13, 822828.

(16) Stadler, P.; Mohamed, S. A.; Gasiorowski, J.; Sytnyk, M.; Yakunin, S.; Scharber, M. C.; Enengl, C.; Enengl, S.; Egbe, D. A. M.; El-Mansy, M. K.; Obayya, S. S. A.; Sariciftci, N. S.; Hingerl, K.; Heiss, W. Iodide-Capped PbS Quantum Dots: Full Optical Characterization of a Versatile Absorber. Adv. Mater. 2015, 27, 15331539.

(17) Woo, J. Y.; Ko, J.-H.; Song, J. H.; Kim, K.; Choi, H.; Kim, Y.-H.; Lee, D. C.; Jeong, S. Ultrastable PbSe Nanocrystal Quantum Dots via in Situ Formation of Atomically Thin Halide Adlayers on PbSe(100). J. Am. Chem. Soc. 2014, 136, 8883-8886.

(18) Sayevich, V.; Gaponik, N.; Plötner, M.; Kruszynska, M.; Gemming, T.; Dzhagan, V. M.; Akhavan, S.; Zahn, D. R. T.; Demir, H. V.; Eychmüller, A. Stable Dispersion of Iodide-Capped PbSe Quantum Dots for High-Performance Low-

Temperature Processed Electronics and Optoelectronics. Chem. Mater. 2015, 27, 4328-4337.

(19) Zhang, Z.; Yang, J.; Wen, X.; Yuan, L.; Shrestha, S.; Stride, J. A.; Conibeer, G. J.; Patterson, R. J.; Huang, S. Effect of Halide Treatments to PbSe Quantum Dot Thin Films: Stability, Hot Carrier Lifetime and Application to Photovoltaics. J. Phys. Chem. C 2015, 119, 24149-24155.

(20) Spitler, M. T.; Parkinson, B. A. Dye Sensitization of Single Crystal Semiconductor Electrodes. Acc. Chem. Res. 2009, 42, 2017-2029.

(21) Laurie A. King; Meghan E. Kern; B. A. Parkinson. Sensitization of Single Crystal Substrates. In Photoinduced Processes at Surfaces and in Nanomaterials; ACS Symposium Series; American Chemical Society, 2015; Vol. 1196, pp 1-45.

(22) Liang, Y.; Novet, T.; Thorne, J. E.; Parkinson, B. A. Photosensitization of ZnO Single Crystal Electrodes with PbS Quantum Dots. Phys. Status Solidi A 2014, 211, 1954-1959. 
(23) Mora-Sero, I.; Gimenez, S.; Moehl, T.; Fabregat-Santiago, F.; Lana-Villareal, T.; Gomez, R.; Bisquert, J. Factors Determining the Photovoltaic Performance of a CdSe Quantum Dot Sensitized Solar Cell: The Role of the Linker Molecule and of the Counter Electrode. Nanotechnology 2008, 19, 7.

(24) CRC Handbook of Chemistry and Physics, 77th Edition, 1996-1997.; CRC Press.

(25) Boschloo, G.; Hagfeldt, A. Characteristics of the Iodide/Triiodide Redox Mediator in Dye-Sensitized Solar Cells. Acc. Chem. Res. 2009, 42, 1819-1826.

(26) Sambur, J. B.; Parkinson, B. A. Size Selective Photoetching of CdSe Quantum Dot Sensitizers on Single-Crystal $\mathrm{TiO}_{2}$. ACS Appl. Mater. Interfaces 2014, 6, 21916-21920.

(27) Jasieniak, J.; Califano, M.; Watkins, S. E. Size-Dependent Valence and Conduction Band-Edge Energies of Semiconductor Nanocrystals. ACS Nano 2011, 5, 5888-5902. 